\title{
Circuito de Instrumentação Monolítico aplicado ao Sensor Magnético \\ Integrado CMOS do tipo Split-Drain
}

\section{Monolithic Implementation of an Instrumentation Circuit Applied to the CMOS Split-Drain Magnetic Sensor}

\author{
Fernando C. Castaldo ${ }^{1}$; Carlos Alberto dos Reis Filho ${ }^{2}$
}

\begin{abstract}
Resumo
Um circuito de instrumentação foi integrado em tecnologia de $0,6 \mathrm{~mm}$ para ser utilizado como amplificador e condicionador de sinal produzido pelo sensor de campo magnético CMOS MAGFET do tipo splitdrain. O circuito emprega uma estrutura de alto ganho que opera no modo realimentado e realiza a conversão corrente-tensão possibilitando a leitura do campo magnético aplicado diretamente na forma de tensão. Medidas da característica estática de transferência, resposta ao campo magnético aplicado, obtenção da resposta em freqüência e análise de ruído permitiram caracterizar a associação sensoramplificador. Suas propriedades gerais tais como sensibilidade e resolução também foram avaliadas.
\end{abstract}

Palavras-chave: MAGFET, Split-Drain, Sensor Magnético

\begin{abstract}
A CMOS instrumentation circuit implemented in $0,6 \mathrm{~mm}$ technology intended for to be used as a signal conditioner applied to the MAGFET split-drain magnetic field sensor transistor is proposed. The circuit features a high gain structure and should be operated in feedback mode, achieving a current-voltage conversion that enables a load to be directly driven by the applied magnetic field. Static transfer characteristic measurements, magnetic field response, bandwidth and noise measurements are carried out in order to evaluate the circuit performance as a magnetic detector.
\end{abstract}

Key words: MAGFET, Split-Drain, Magnetic Sensor, Transresistance.

Mestre e Doutor em Engenharia Elétrica na área de Sensores, Instrumentação Eletrônica e Microeletrônica. Atualmente Prof. Adjunto A no Departamento de Eng. Elétrica da Universidade Estadual de Londrina

2 Mestre e Doutor em Engenharia Elétrica na área de Sensores, Instrumentação Eletrônica e Microeletrônica. Prof. Adjunto no Departamento de Instrumentação, Semicondutores e Fotônica da Faculdade de Engenharia Elétrica da Universidade Estadual de Campinas 


\section{Introdução}

Atualmente, sensores magnéticos integrados apresentam grande interesse na implementação de sistemas on-chip aplicados na detecção de campos magnéticos em sistemas de posicionamento, medição de corrente, leitura de dados, entre outros. Devido à compatibilidade dos sensores magnéticos MAGFET do tipo split-drain (POPOVIC, 1997) com a tecnologia CMOS, o próprio elemento sensor e os circuitos de instrumentação necessários para o condicionamento do sinal podem ser construídos monoliticamente. Apesar de os sensores de campo magnético CMOS apresentarem desempenho inferior quando comparados aos implementados em outras tecnologias, a possibilidade de integração permite construir micro-sensores competitivos. Esse fato tem motivado a indústria e a academia, e diversos trabalhos têm sido divulgados, inclusive bastante recentes (LI et al., 2005). A literatura menciona uma grande diversidade de circuitos de instrumentação direcionados a detectores magnéticos e aplicações, basicamente voltados à detecção de campos magnéticos estáticos. Em (HABERLI et al., 1995), é apresentado um medidor vetorial de campo magnético em duas dimensões (2-D), o qual utiliza um array de transistores laterais disponíveis no processo CMOS (LTM-CMOS). Um sensor 3-D implementado com transistores bipolares verticais associados a um transistor split-drain é citado em (ZHANG; MISRA, 1991). Uma aplicação integrada para medição de corrente utilizando um dispositivo Spinning Hall com interface serial é apresentada em (MALCOVATI; MALOBERTI, 1999) enquanto que uma proposição para medição de corrente em circuitos integrados Smart-Power CMOS foi discutida em (BUSATTO et al., 2003). Osciladores controlados por indução magnética também têm sido reportados (DOYLE; LYDEN, 1994) e (NATHAN, 1987). De uma forma geral, todas as implementações citadas são susceptíveis a offset (sistemáticos ou devido a mismatching) e ruído, principalmente do tipo $1 /$ f nos detectores de campo magnético estático. Outros problemas, como deriva térmica, variação dos parâmetros de processo, etc. também afetam de modo importante o desempenho destes sensores. Assim, é necessário a inclusão de mecanismos de compensação que permitam ajustes do circuito pósfabricação ou utilizar-se de estruturas que possibilitem a operação com ganho ajustável bem como prever circuitos de correção e ajuste. Com este intuito, um circuito detector de campo magnético baseado no transistor split-drain CMOS (transistor e instrumentação associada) é implementado e verificado experimentalmente. As características básicas de detecção e relação sinal-ruído são investigadas com o objetivo de se determinar a sua sensibilidade e resolução respectivamente.

\section{Circuito Proposto: Amplificador de Transresistência}

Considerando-se a operação em modo corrente de um dispositivo split-drain (POPOVIC, 1991), o circuito de instrumentação associado poderia ser realizado a partir do emprego de um amplificador de transresistência, que converte diretamente em uma tensão a corrente diferencial gerada pelo split-drain sob a ação de um campo magnético. Essa tensão pode ser processada pelas etapas seguintes do sistema, tais como os circuitos de leitura e, por esta razão, a literatura sugere a utilização de amplificadores magneto-operacionais (MOP) que realizam diretamente a conversão da corrente diferencial do transistor em uma tensão de saída. Transistores split-drain conectados a amplificadores diferenciais totais (Fully-Differential) do tipo folded-cascode proporcionam elevados ganhos de conversão corrente-tensão, sendo necessário o emprego de uma estrutura de realimentação para estabilização do ganho de modo comum. Esse circuito 
de realimentação pode ser realizado com capacitores chaveados (CHEN, 2003) ou operar em modo contínuo (LIU, 1999). Outras implementações de MOPs (também citados como MAGAMP) têm o ganho de realimentação configurado a partir de resistores externos (RYAN et al., 1992). Partindose de uma sugestão apresentada em (BUSATTO et al., 2003) implementou-se um amplificador de transresistência em tecnologia CUB06 $6^{3}$, o qual não emprega rede de resistores para ajuste do ganho, pois o mesmo é configurável a partir de uma corrente de polarização. Esta implementação é a seguir apresentada.

Trata-se basicamente de um circuito amplificador diferencial com entrada em corrente e sinal de realimentação também em corrente. $O$ sinal gerado pelo transistor split-drain sob a ação de um campo magnético é amplificado e disponibilizado na saída do amplificador (Vo). Basicamente, o transistor splitdrain conduz uma corrente DC $\left(2 . \mathrm{I}_{\mathrm{DC}}\right)$ que se divide igualmente entre os drenos $\left(\mathrm{I}_{\mathrm{DC}}\right)$. Superposta a esta corrente contínua, uma corrente diferencial $\left(\mathrm{I}_{\mathrm{IN}}\right)$ é produzida por ação de Lorentz quando um campo magnético incide perpendicularmente ao dispositivo, conforme indicado na Fig. 1.

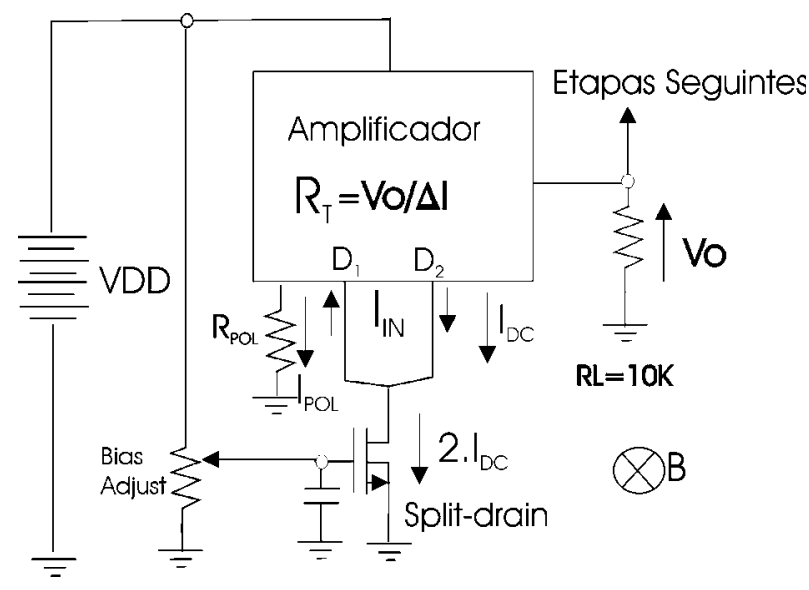

Figura 1. Sensor Magnético Split-Drain conectado ao Amplificador de Transresistência implementado em tecnologia CUB06
O circuito do amplificador de transresistência implementado é indicado na Fig.2. A corrente diferencial de entrada $\left(\mathrm{I}_{\mathrm{IN}}\right)$ percorre um caminho de baixa resistência, sendo então subtraída em um espelho-N tipo cascode. A resistência de saída (Ro) dos espelhos cascode (M1,M2) e (M3,M4) realiza a conversão da corrente diferencial em uma tensão (Vo) que é aplicada ao próximo circuito, um conversor tensão-corrente, por meio do transistor M6, produzindo a corrente de saída (Io). Esse transistor tipo $\mathrm{P}$ atua como um follower, direcionando o sinal de tensão diretamente sobre a condutância de saída do transistor M5 que produzirá a corrente de dreno em M6, que será espelhada.

Esta corrente, sendo espelhada, estabelece a corrente de erro (Io), que é subtraída da corrente de entrada $\left(\mathrm{I}_{\mathrm{IN}}\right)$, fechando a malha de realimentação negativa. Sendo a condutância de M5 dependente da corrente de polarização, ajustável através de $\mathrm{R}_{\mathrm{POL}}$, um controle de feedback é estabelecido. A partir da quantidade de realimentação, o ganho de conversão, ou a transresistência pode ser variada. Adicionalmente, um driver do tipo follower composto de um par de transistores $\mathrm{P}$ e $\mathrm{N}$ são conectados na saída do circuito para se evitar o carregamento da resistência de saída dos espelhos. O sensor magnético do tipo split-drain é conectado nos pontos D1 e D2. A partir das tensões e correntes indicadas na Fig.1, a transresistência pode ser determinada de acordo com (1).

$$
\frac{V o}{I_{I N}}=R_{T}=\frac{2 . R o}{1+R o \cdot g_{d s} 5}
$$

Em (1), os parâmetros referem-se à resistência de saída $\left(\mathrm{R}_{\mathrm{O}}\right)$ combinada dos espelhos cascode (M1, M2) e (M3,M4) e à condutância dreno-fonte do transistor MOS M5 $\left(\mathrm{g}_{\mathrm{ds} 5}\right)$. Os demais parâmetros podem ser determinados a partir das relações apresentadas em (2) e, em conformidade com os

3 CUB06 refere-se a uma tecnologia proprietária da AustriaMicrosystems em 0.6mm. 


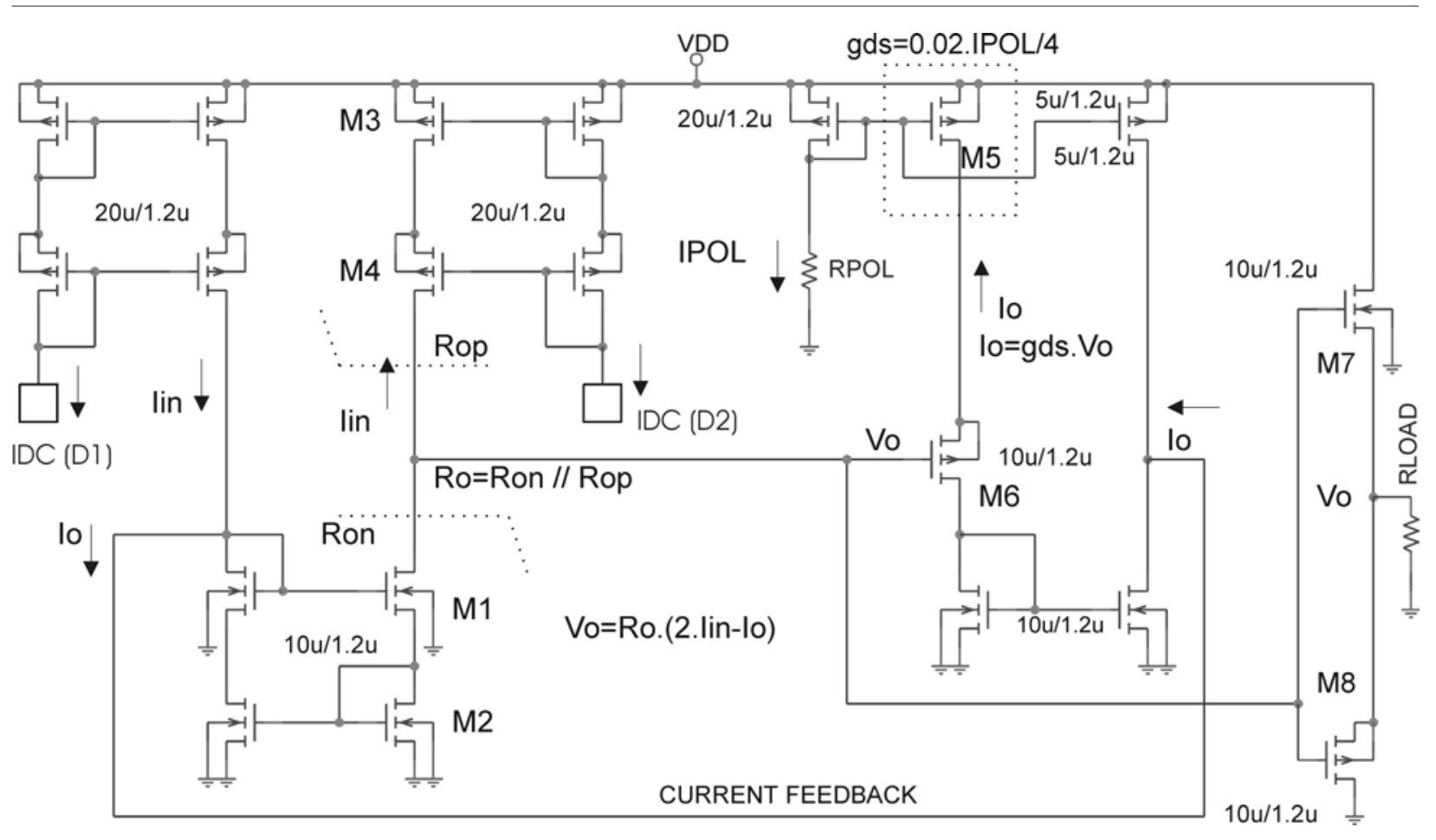

Figura 2. Amplificador de Transresistência implementado em tecnologia CUB06

parâmetros da tecnologia utilizada CUB06: $\mathrm{r}_{\mathrm{ds}}$, resistência dreno-source do transistor considerado, $\mathrm{g}_{\mathrm{m}}$ a respectiva transcondutância, $\lambda$ o fator de modulação de canal (transistor MOSP, $\lambda_{\mathrm{P}}=0,02$, $\left.\operatorname{MOSN}, \lambda_{\mathrm{N}}=0,01\right)$, g o coeficiente de efeito de corpo, $\gamma_{\mathrm{N}}=0,8 \sqrt{ } \mathrm{V}, \gamma_{\mathrm{P}}=0,48 \sqrt{ } \mathrm{V}, \phi_{0}=2 . \phi_{\mathrm{F}}=0,848 \mathrm{~V}$ e $\mathrm{V}_{\mathrm{BS}}$ a tensão body-source do transistor (GEIGER; ALLEN; STRADER, 1990).

$$
\begin{aligned}
& R o=\text { Ron } / / \text { Rop } \quad g_{d s 5}=\lambda_{P} \frac{I_{P O L}}{4} \\
& R o n=r_{d s 1}+r_{d s 2}+g_{m 1} \cdot r_{d s 1} \cdot r_{d s 2} \cdot\left(1+\frac{\gamma}{2 \cdot \sqrt{\phi_{0}-V_{B S}}}\right) \\
& R o p=r_{d s 3}+r_{d s 4}+g_{m 3} \cdot r_{d s 3} \cdot r_{d s 4} \\
& r_{d s}=\left(\lambda \cdot I_{D}\right)^{-1} \quad \lambda_{P}=0,02 \quad \lambda_{N}=0,01
\end{aligned}
$$

O ganho de conversão do circuito (Transresistência) pode ser determinado a partir de (1) e (2), de acordo com o procedimento adotado em (GEIGER; ALLEN; STRADER, 1990), mas o valor de transresistência fica superestimado, uma vez que a determinação da condutância entre dreno e fonte dos transistores é apenas aproximada. A determinação exata das condutâncias dos transistores em função da corrente estática de entrada produzida pelo transistor split-drain $\left(\mathrm{I}_{\mathrm{DC}}\right)$ e de polarização $\left(\mathrm{I}_{\mathrm{POL}}\right)$ pode ser realizada através de simulação em Pspice baseada nos parâmetros da tecnologia. Esse procedimento produz melhores resultados. Na Fig.3 estão representados alguns resultados de simulação para o ganho (transresistência) do circuito em função da corrente estática em cada entrada $\left(\mathrm{I}_{\mathrm{DC}}\right)$, parametrizada na corrente de polarização $\left(\mathrm{I}_{\mathrm{POL}}\right)$, que determina o ganho do circuito. 


\section{Trans-Resistencia}

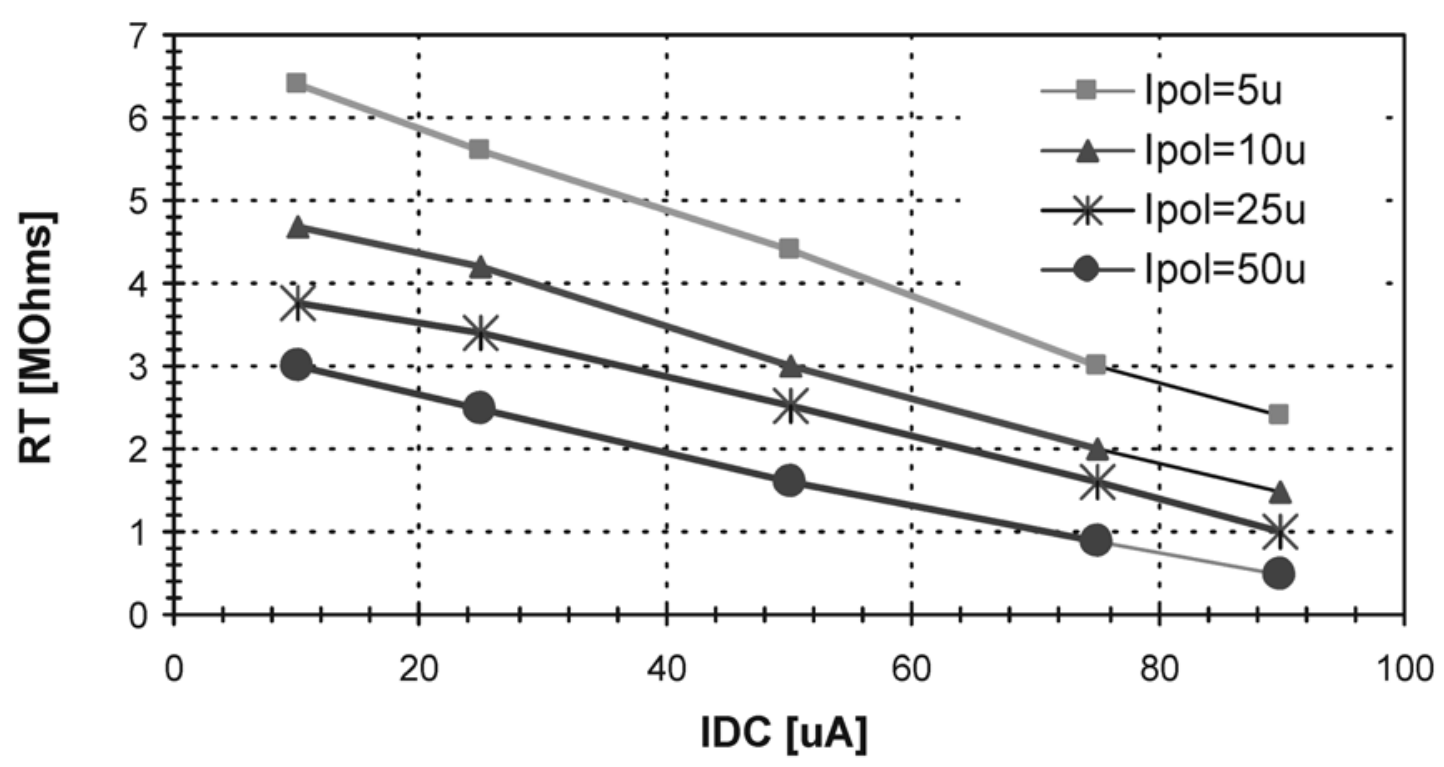

Figura 3. Ganho do Amplificador ou Transresistência obtido por simulação

Uma verificação da estabilidade do circuito pode ser realizada a partir de uma simulação em malha aberta como indicado na Fig.4. A simulação é realizada, considerando uma condição de operação
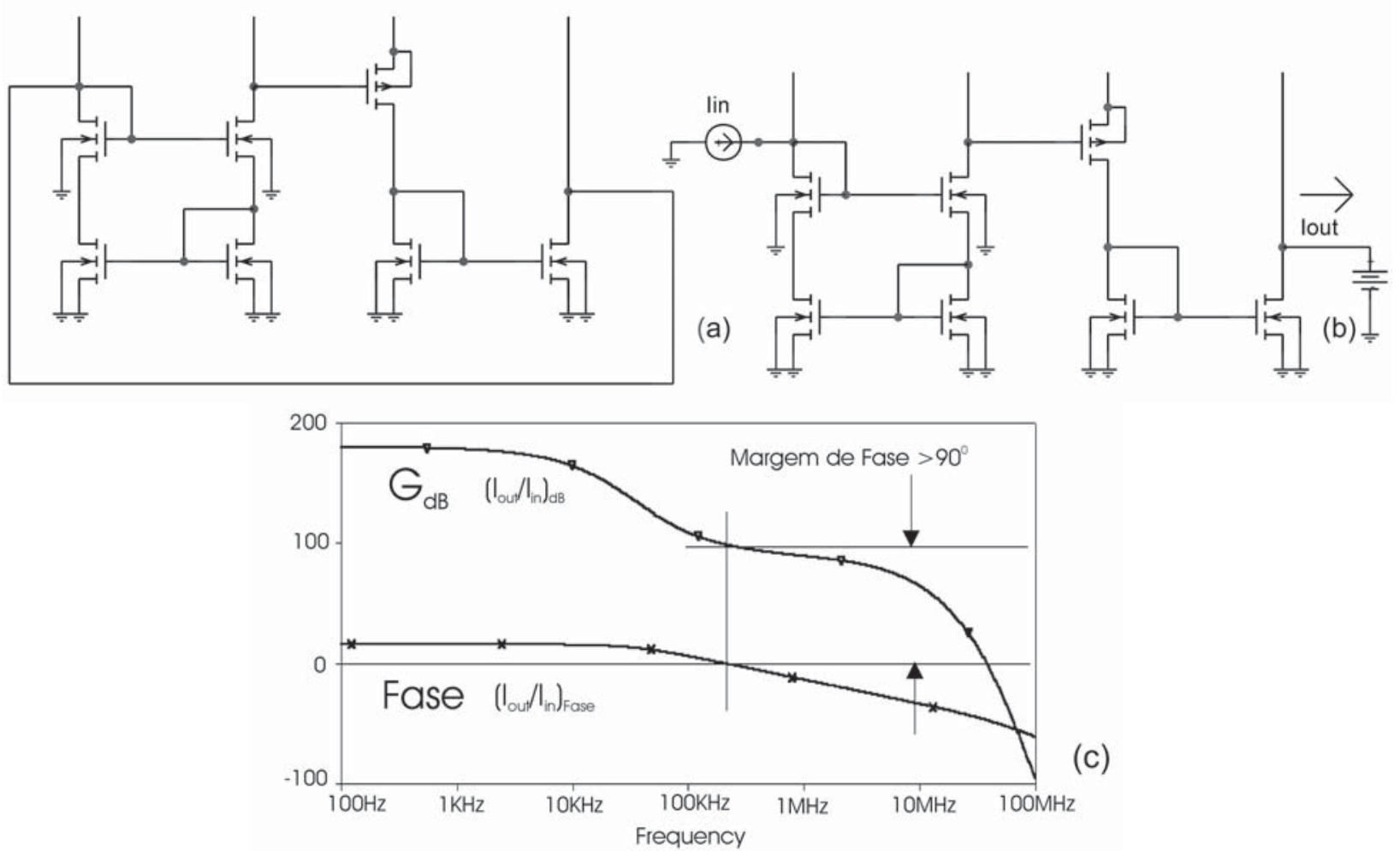

Figura 4. (a) Circuito em malha fechada original. (b) Obtenção da malha aberta. (c) Resposta em freqüência da malha aberta (Simulação) 
em que o ganho é máximo $\left(\mathrm{I}_{\mathrm{POL}}=5 \mu \mathrm{A}\right.$ e $\mathrm{I}_{\mathrm{DC}}=10 \mu \mathrm{A}$ em cada entrada), e, com isso, se busca garantir a estabilidade em toda a faixa de operação. O ponto de realimentação de corrente apresenta baixa impedância (impedância de entrada do espelho cascode), logo na abertura da malha, representa-se este efeito por uma fonte de tensão com valor suficiente para polarizar o transistor de saída. No ponto de entrada da realimentação, acrescenta-se uma fonte de corrente, representando a elevada impedância de saída do circuito conversor tensãocorrente. Como o sinal de realimentação é corrente, a resposta em freqüência do ganho de malha aberta é definida como $\mathrm{I}_{\mathrm{OUT}} / \mathrm{I}_{\mathrm{IN}}$, dado em $\mathrm{dB}$. Observa-se que o sistema é estável, apresentando um margem de fase igual ou superior a $90^{\circ}$, para qualquer condição de operação do amplificador. $O$ circuito foi simulado com base nos parâmetros da tecnologia CUB06.

\section{Resultados Experimentais}

O circuito implementado foi testado por meio de medidas estáticas para verificação do ganho, resposta ao campo magnético aplicado, resposta em freqüência e determinação do ruído gerado. $\mathrm{Na}$ seqüência, são detalhados os procedimentos realizados.

Característica Estática:

Desconectando-se o transistor split-drain das entradas do amplificador (pontos D1 e D2), o ganho ou transresistência pode ser avaliado por meio de medidas estáticas realizadas no Analisador de Parâmetros de Semicondutores HP4155. Para tal, utilizando-se as fontes de excitação do próprio equipamento, polariza-se o circuito e aplica-se uma variação de corrente diferencial na entrada. Como pode ser observado na Fig.5, por meio da característica de saída $\mathrm{V}_{\mathrm{O}} / \mathrm{I}_{\mathrm{IN}}$, transresistência pode ser determinada a partir da inclinação da reta tangente, em função da corrente de polarização $\left(\mathrm{I}_{\mathrm{POL}}\right)$. Como previsto nas Eqs. (1) e (2), a corrente de polarização permite um ajuste do ganho do amplificador. No exemplo em questão, adotou-se uma corrente de entrada $\left(\mathrm{I}_{\mathrm{DC}}\right)$ de $10 \mu \mathrm{A}$ mas outros valores podem ser igualmente aplicados. Verifica-se uma razoável correspondência com o valor da transresistência obtida a partir da Fig.3.

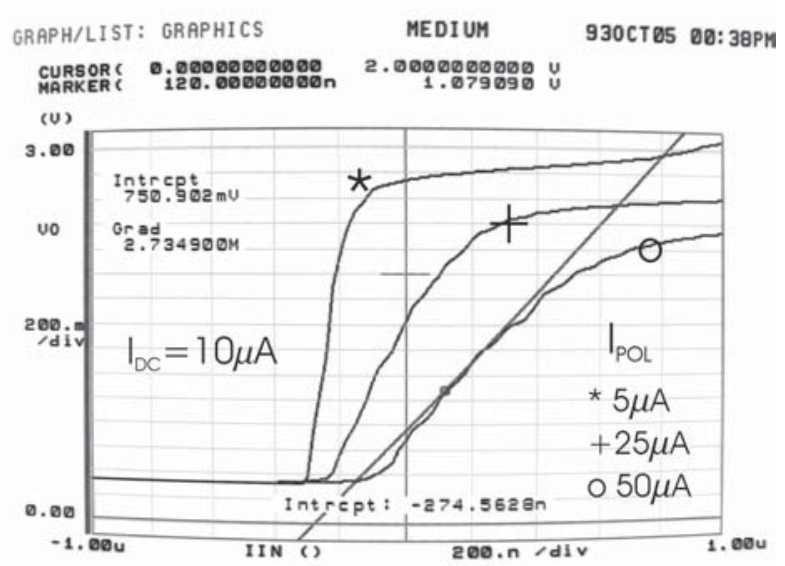

Figura 5. Característica estática levantada no HP4155 e determinação da Transresistência

Detecção Magnética:

Ensaios com campos magnéticos aplicados permitem verificar a resposta do detector. O campo magnético é gerado a partir de um solenóide previamente calibrado, e aplicado diretamente sobre o circuito integrado, conforme indicado na Fig.6(a). Dessa forma, determina-se a característica da tensão de saída em função do campo magnético aplicado. No ensaio realizado, aplicou-se um campo magnético local com freqüência de $100 \mathrm{~Hz}$ e amplitude variável de até $6 \mathrm{mT}$ (RMS). O solenóide é conectado a um gerador de funções HP33120 que determina a amplitude e freqüência do campo produzido.

Fixando-se a polarização do transistor split-drain em $V_{G}=3,2 V, \quad I_{D}=190 \mu A \quad\left(I_{D C}=95 \mu \mathrm{A}\right)$ e do amplificador em $\mathrm{I}_{\mathrm{POL}}=5 \mu \mathrm{A}, \mathrm{R}_{\mathrm{L}}=10 \mathrm{~K} \Omega$, aplica-se o campo magnético e realiza-se a leitura do sinal detectado no analisador de sinais HP3562A. Nessas 
condições de operação, o ganho do amplificador pode ser determinado a partir da Fig.3 e vale aproximadamente $\mathrm{R}_{\mathrm{T}}=2.65 \times 10^{6} \Omega$. Para o transistor split-drain conectado ao amplificador, a sensibilidade é $\mathrm{S}=2,6 \% / \mathrm{T}$. Considerando que o mesmo opera com corrente de polarização $\mathrm{I}_{\mathrm{D}}=190 \mu \mathrm{A}$, a corrente diferencial produzida por um campo magnético aplicado pode ser obtida a partir de $\mathrm{I}_{\mathrm{IN}}=\mathrm{S} . \mathrm{I}_{\mathrm{D}}$. B . Substituindo valores, obtém-se. $\mathrm{I}_{\mathrm{IN}}=4.94 \times 10^{-6} \times \mathrm{B}$.
Essa corrente produzirá uma tensão de saída correspondente a $\mathrm{V}_{0}=\mathrm{R}_{\mathrm{T}} \cdot \mathrm{I}_{\mathrm{IN}}$, onde $\mathrm{R}_{\mathrm{T}}$ refere-se ao ganho de transresistência do amplificador. Substituindo a expressão para $\Delta \mathrm{I}$, obtém-se $\mathrm{V}_{0}=2.65 \times 10^{6} \times 4.94 \times 10^{-6} \times \mathrm{B}$, ou $\mathrm{V}_{0} / \mathrm{B}=13.1$ que corresponde ao coeficiente da reta ajustada às medidas em $100 \mathrm{~Hz}$ conforme indicado na Fig.6(b). Observa-se uma característica essencialmente linear da tensão de saída com a intensidade do campo magnético aplicado.
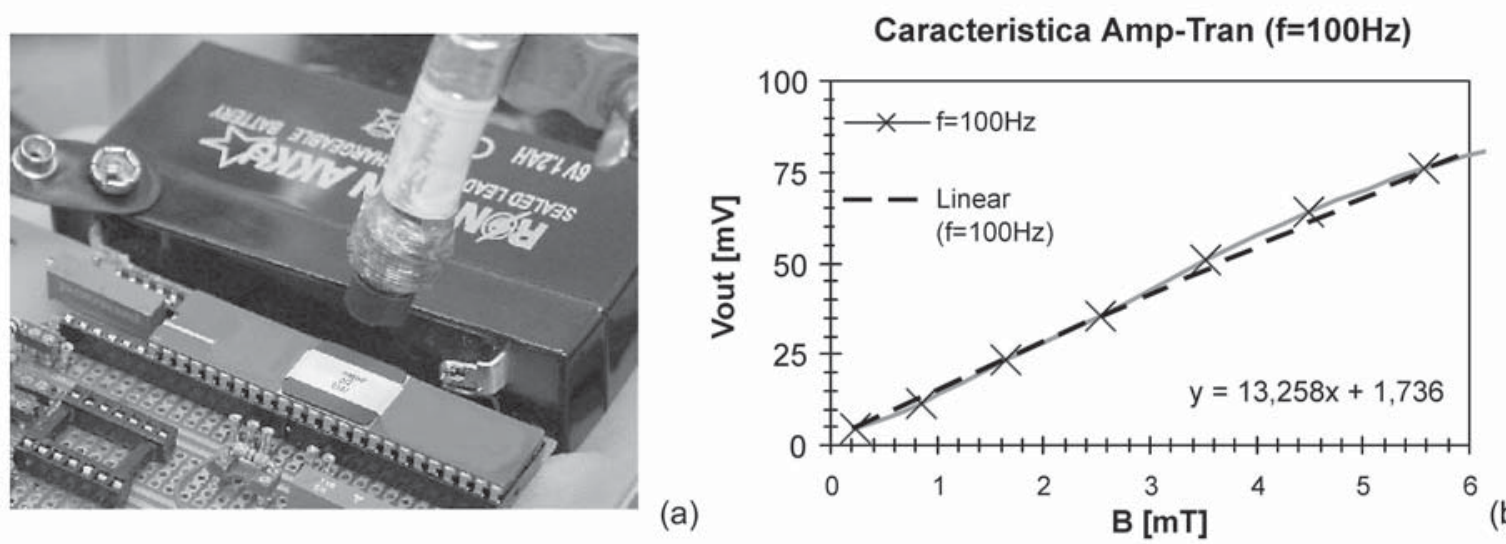

(b)

Figura 6. (a) Aplicação do campo magnético. (b) Resposta do circuito detector a um campo magnético aplicado de $100 \mathrm{~Hz}$

\section{Resposta em freqüência:}

A resposta em freqüência de malha fechada do circuito com o transistor split-drain desconectado foi levantada experimentalmente no analisador de redes (network analyser-HP3577) para uma corrente de polarização $\mathrm{I}_{\mathrm{POL}}=50 \mu \mathrm{A}$ e corrente de entrada $\mathrm{I}_{\mathrm{DC}}=50 \mu \mathrm{A}$. O setup de conexão ao HP3577 é indicado na Fig.7(a). Dois resistores externos $\mathrm{R}_{\mathrm{IN}}=47 \mathrm{~K} \Omega$ foram utilizados para se polarizar cada uma das entradas do amplificador com cerca de $\mathrm{I}_{\mathrm{DC}}=50 \mu \mathrm{A}$. Um resistor de carga de $\mathrm{R}_{\mathrm{L}}=10 \mathrm{~K} \Omega$ foi conectado à saída do amplificador. $\mathrm{O}$ sinal de tensão gerado pelo HP3577 com freqüência variável é aplicado a uma das entradas do circuito e também à entrada de referência $\mathbf{R}$ do equipamento, cuja impedância de entrada foi ajustada em $50 \Omega$. O sinal de tensão gerado pelo amplificador é aplicado à

entrada $\mathbf{A}$ do analisador de redes, com impedância de entrada ajustada em $1 \mathrm{M} \Omega$ para não carregar o circuito de saída do amplificador de transresistência. A amplitude do sinal pode ser variada entre $1 \mathrm{mV}$ e $10 \mathrm{mV}$ de acordo com a melhor visualização da resposta. A saída é apresentada como a razão logarítmica entre o sinal de saída do amplificador aplicado na entrada $\mathbf{A}$ e o sinal gerado pela fonte aplicado na entrada $\mathbf{R}$, dado em $\mathrm{dB}$. Observa-se que a resposta em freqüência é limitada em dezenas de $\mathrm{KHz}$ devido às capacitâncias dos pads do circuito integrado como indicado na Fig.7(b). A utilização de buffers nos pontos de saída do circuito amplia significativamente a banda de resposta em freqüência do amplificador, como verificado posteriormente através de simulações. 

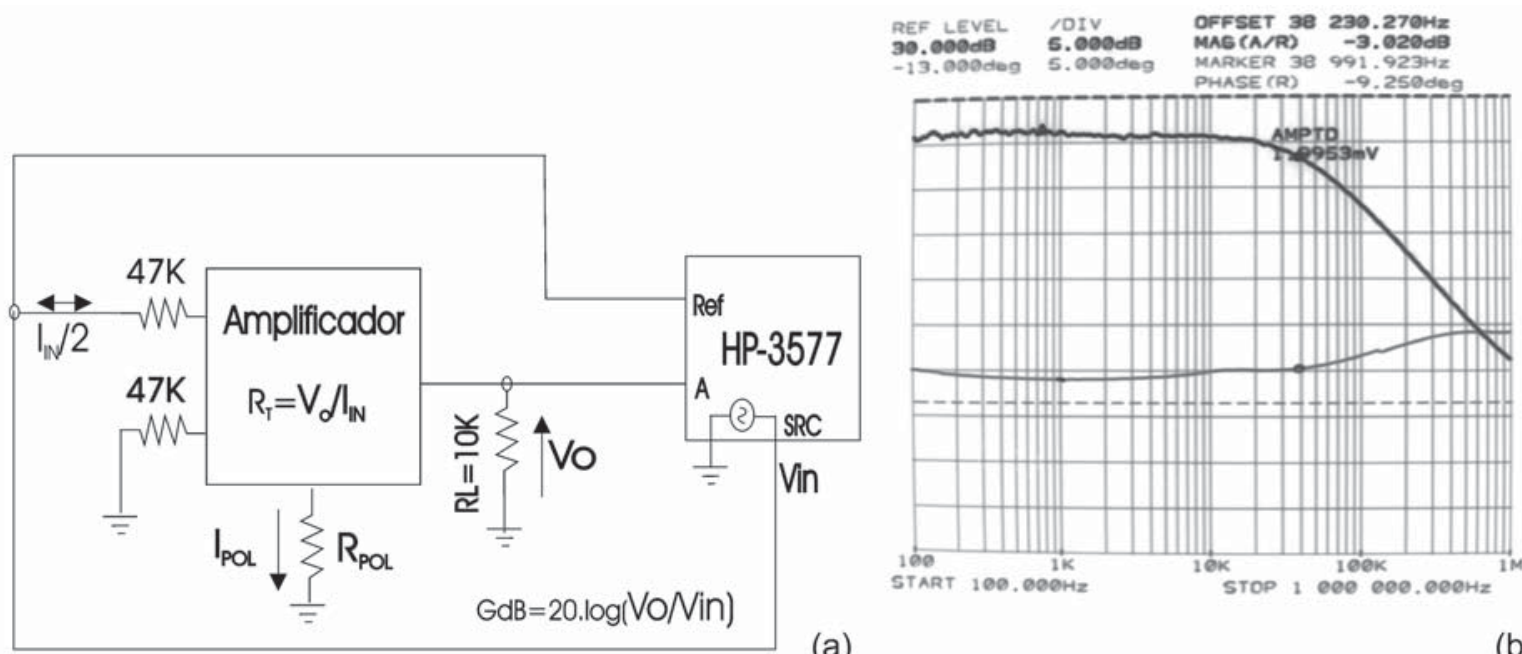

(a)

(b)

Figura 7. (a) Setup utilizado para verificação da resposta em freqüência. (b) Resultados obtidos.

\section{Resolução do Detector Magnético}

A resolução do sensor pode ser avaliada a partir do nível de ruído gerado pelo circuito. Ajustando-se as condições de polarização do circuito como anteriormente, $\mathrm{V}_{\mathrm{G}}=3.2 \mathrm{~V}, \mathrm{I}_{\mathrm{DC}}=95 \mu \mathrm{A}, \mathrm{I}_{\mathrm{POL}}=5 \mu \mathrm{A}$, $\mathrm{R}_{\mathrm{L}}=10 \mathrm{~K} \Omega$, aplicou-se um campo magnético de $220 \mu \mathrm{T}$ e freqüência $\mathrm{f}=2.5 \mathrm{kHz}$ sobre o circuito integrado. O sinal de saída pode ser observado no analisador de sinais HP3562A, conforme indicado na Fig.8. Para o transistor split-drain-N implementado operando com $\mathrm{I}_{\mathrm{D}}=190 \mu \mathrm{A}$, a corrente diferencial produzida pelo campo magnético $(\mathrm{B}=220 \mu \mathrm{T})$ será $\mathrm{I}_{\mathrm{IN}}=\mathrm{S} \cdot \mathrm{I}_{\mathrm{D}} \cdot \mathrm{B}$ ou $\mathrm{I}_{\mathrm{IN}}=1.1 \times 10^{-9} \mathrm{~A}$. Esta corrente produzirá uma tensão de saída correspondente a $\mathrm{V}_{0}=\mathrm{R}_{\mathrm{T}} \cdot \mathrm{I}_{\mathrm{IN}}$, onde $\mathrm{R}_{\mathrm{T}}$ refere-se ao ganho de transresistência do amplificador que nesta condição de operação, $\mathrm{R}_{\mathrm{T}}=2.65 \mathrm{M} \Omega$ como anteriormente. A tensão RMS lida no analisador de sinal será $\mathrm{V}_{0}=\mathrm{R}_{\mathrm{T}} \cdot \mathrm{I}_{\mathrm{IN}}=2.8 \mathrm{mV}_{\mathrm{RMS}}$. Considerando uma resolução de banda de $12.5 \mathrm{~Hz}$ (BW-Resolution Bandwidth) utilizada pelo analisador de sinal na freqüência de $2.5 \mathrm{KHz}$, a tensão de saída será visualizada no analisador de sinais como $2.8 \times 10^{-3} /$ $\sqrt{ } 12.5=790 \mu \mathrm{V} / \sqrt{ } \mathrm{Hz}$. Observa-se, neste caso, que o campo mínimo detectável equivale aproximadamente a $250 \mu \mathrm{V} / \sqrt{ } \mathrm{Hz}$, ou em valores RMS absolutos para $1 \mathrm{~Hz}$ de BW: $\mathrm{Vo}=250 \mu \mathrm{V} / \sqrt{ } \mathrm{Hz} . \sqrt{ } 12.5=880 \mu \mathrm{V}$. Projetando-se para a entrada do circuito este valor, obtém-se: $\mathrm{I}_{\mathrm{IN}}=\mathrm{V}_{0} / \mathrm{R}_{\mathrm{T}}=330 \mathrm{pA}$ e aplicando-se $\mathrm{I}_{\mathrm{IN}}=\mathrm{S} . \mathrm{I}_{\mathrm{D}} \cdot \mathrm{B}_{\mathrm{MIN}} \operatorname{logo}$, para as mesmas condições de operação $\mathrm{B}_{\mathrm{MIN}}=70 \mu \mathrm{T}(\mathrm{Em} 1 \mathrm{~Hz}$ de banda). Este campo magnético mínimo corresponde à resolução alcançada pelo detector. 


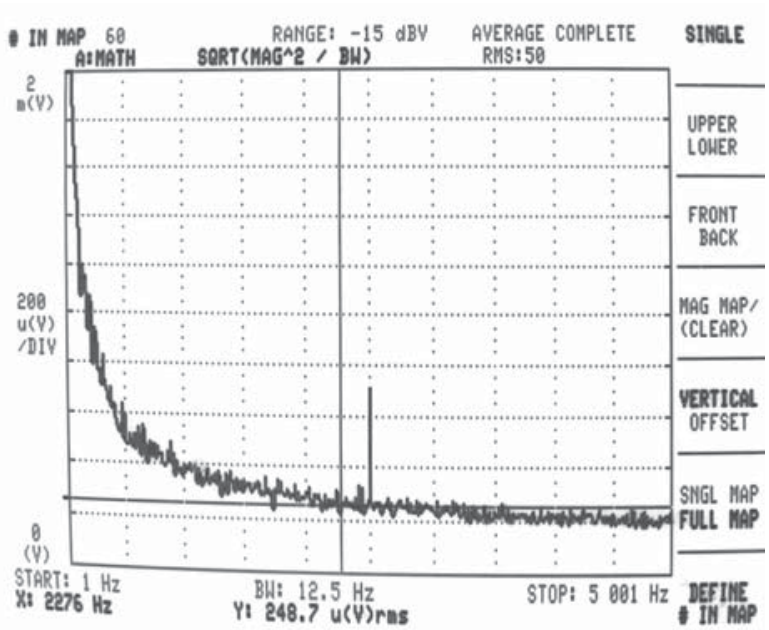

Figura 8. Detecção de um campo de $220 \mathrm{mT}$ e ruído de fundo associado

Na Fig.9 é apresentada a fotomicrografia do circuito implementado em CUB06. Identifica-se o transistor split-drain como sendo a maior estrutura.

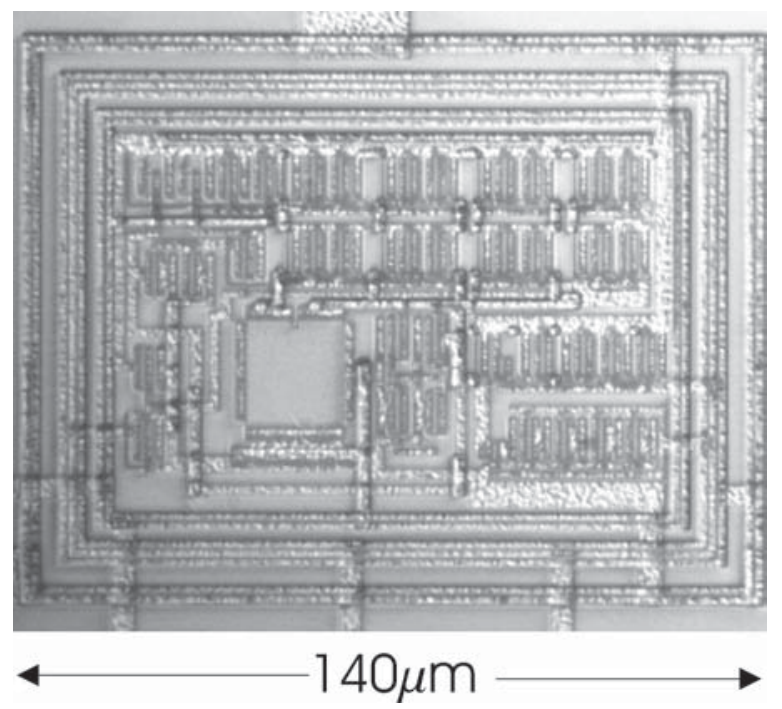

Figura 9. Fotomicrografia do circuito detector magnético implementado em CUB06

\section{Conclusões}

Um circuito detector magnético foi implementado em tecnologia CUB06 baseado no sensor CMOS MAGFET do tipo split-drain. Como o sinal produzido por este sensor é da ordem de pA a nA, é necessário o condicionamento do sinal captado. Assim, este circuito atua como amplificador e condicionador de sinal para as etapas posteriores de uma determinada aplicação. O amplificador concebido baseia-se em uma estrutura de alto ganho que opera no modo realimentado e realiza a conversão corrente-tensão através dos próprios elementos do circuito. A caracterização do detector foi realizada com base na determinação da característica de transferência estática, resposta ao campo aplicado, obtenção da resposta em freqüência e análise de ruído. A metodologia de projeto sugerida baseou-se no tratamento clássico dado às equações que determinam a condutância de saída de um transistor MOS. Entretanto, a análise a partir de simulação em PSPICE permitiu obter resultados mais consistentes com os obtidos experimentalmente, devido à utilização de modelos mais complexos (BSIM) baseados nos parâmetros da tecnologia utilizada. Este circuito apresenta a vantagem de entregar o sinal monitorado diretamente na forma de tensão, o que é bastante desejável em certas aplicações.

\section{Agradecimentos}

CAPES pelo suporte financeiro na forma de bolsa PICDT.

\section{Referências}

BUSATTO, G.; LA CAPRUCCIA, R.; IANNUZZO, F.; VELARDI, F.; RONCELLA, R.; BUSATTO, G. Magfet based current sensing for power integrated circuit. Microelectronics Reliability, London, v.43, p.577-583, 2003. 
CHEN, S. L.; KUO, C.H.; LIU, S. U. CMOS Magnetic Field to frequency converter. IEEE Sensors Journal, Madison, v.3, n.2, p.241-245, Apr. 2003.

DOYLE, J.; LYDEN, C. High sensitivity, low power, silicon manetic field detector. In: IEEE CUSTOM INTEGRATED CIRCUITS CONFERENCE, 1994. Proceeding... San Diego: Nat. Microelectron. Res. Centre, 1994. p.275-277.

GEIGER, R.; ALLEN, P.; STRADER, N. VLSI-Design techniques for analog and digital circuits. New York: McGraw, 1990. (Hill Series in Materials Science and Engineering)

HABERLI, A.; MALCOVATI, P.; SCHNEIDER, M.; CASTAGNETTI, R.;. BALTES, H. Contactless angle measurement by CMOS magnetic sensor with on-chip read-out circuit. In: INTERNATIONAL CONFERENCE ON SOLID-STATE SENSORS AND ACTUATORS, 8., 1995, Stockholm. Proceeding... Stockholm: Congrex, 1995.

LI, Z.Q.; SUN, X. W.; TAN, S. C.; LI, C. M. A magnetic field-induced current-modulating opamp based on CMOS differential Tesla-Volt multiplier cell for MAGFET 1/f noise reduction. Sensors and Actuators A, v.118, n.2, Feb. 2005.

LIU, S.I.; WEI, J. F.; SUNG, G. M. SPICE Macro Model for MAGFET and its Applications. IEEE Transactions on Circuits and Systems II: Analog and Digital Signal Processing, v.46, n.4, p.370-375, Apr. 1999.
MALCOVATI, P.; MALOBERTI, F. A fully integrated CMOS magnetic current monitor. PROCEEDINGS OF IEEE INTERNATIONAL SYMPOSIUM ON CIRCUITS AND SYSTEMS-ISCAS. v.5, p.128-131,1999.

NATHAN, A .; MCKAY, I. A.; FILANOVSKY, I. M. Design of a CMOS Oscilattor with Magnetic-Fiield Frequency Modulation. IEEE Journal of solid-State Circuits, v.22, n.2, p.230-232. Apr. 1987.

POPOVIC, R.S. Hall devices for magnetic sensor microsystems. In: INTERNATIONAL CONFERENCE ON SOLID-STATE SENSORS AND ACTUATORS, 8., 1997, Chigago, Proceeding... Chigago: Congrex, 1997. v.1, p.377-380.

POPOVIC, R.S. Hall effect devices: magnetic sensors and characterization of semiconductors. Bristol : IOP Publishing, 1991. (The Adam Hilger series on sensors)

RYAN, J.; DOYLE, J.; BUCKLEY, M.; FLYN, M. A magnetic Field Sensitive amplifier with temperature compensation. IEEE INTERNATIONAL SOLID-STATE CIRCUITS CONFERENCE, p.124-125, 1992.

ZHANG, M; MISRA, D. A Novel 3D Magnetic Field Sensor in Standard CMOS Technology. Digest of Technical Papers. In: INTERNATIONAL CONFERENCE ON SOLID STATE SENSORS AND ACTUATORS. Transducer's 91. p.1085-1088, 1991. 